\title{
Soft topological objects in topological media
}

\author{
Jukka I. Väyrynen ${ }^{* 1)}$ and G.E. Volovik ${ }^{*+2)}$ \\ * Low Temperature Laboratory, Aalto University School of Science, P.O. Box 15100, FI-00076 AALTO, Finland \\ + Landau Institute for Theoretical Physics RAS, Kosygina 2, 119334 Moscow, Russia
}

Submitted January, 2011

\begin{abstract}
Topological invariants in terms of the Green's function in momentum and real space determine properties of smooth textures within topological media. In space dimension $\mathrm{d}=1$ the topological invariant $N_{3}$ in terms of the Green's function $\mathcal{G}\left(\omega, k_{x}, x\right)$ determines the fermion number of the kink, while in space dimension $\mathrm{d}=3$ the topological invariant $N_{5}$ in terms of the Green's function $\mathcal{G}\left(\omega, k_{x}, k_{y}, k_{z}, z\right)$ determines quantization of Hall conductivity in the soliton plane within the topological insulators.
\end{abstract}

PACS:

\section{INTRODUCTION}

In the fully gapped topological systems in even space 'dimension, the Chern-Simons terms are well defined. The prefactors of these terms are represented by topo'logical invariants in momentum-frequency space, which 'are expressed in terms of the Green's function and are 'robust to interactions (see, e.g., Refs. 1, 2, 3, 4, 5, 6] 'for $2+1$ systems; Refs. [6, 7] for $4+1$ systems; and Refs. '8, 9 for systems in general $2 n+1$ spacetime). These invariants determine quantization of the parameters of the system, such as intrinsic Hall and spin-Hall conductivity in $2+1$ and $4+1$ systems. For odd space dimension the situation is more complicated, especially for time reversal invariant (TRI) systems, where the suggested Chern-Simons term formally violates the time reversal symmetry, see, e.g., discussion in Ref. [10. Here we show that though these terms are not welldefined for bulk topological matter, they well describe the properties of smooth textures within the topological matter. The well-defined topological invariants in momentum-frequency space are expressed in terms of 'the Green's function and robust to interactions. They also determine the quantization of the parameters of the 'system, in a given case these are the quantum numbers of the texture, such as the fermion number in $1+1$ system and quantized Hall or spin-Hall conductivity within the smooth interface in the $3+1$ systems.

The smooth textures are analogues of the topological solitons in condensed matter systems with spontaneously broken symmetry. There are two types of topological objects in these condensed matter systems: singular topological defects (such as domain walls, quantized vortices, hedgehogs and dislocations) and continuous structures called topological solitons, skyrmions

\footnotetext{
2) e-mail:jukka.vayrynen@helsinki.fi

2) e-mail: volovik@boojum.hut.fi
}

and textures. As distinct from the singular topological defects, which are described by conventional homotopy groups, textures do not have singularities in the order parameter fields and are described by relative homotopy groups (RHG), see [11. These homotopy groups $\pi_{n}(R, \tilde{R})$ deal with two different manifolds of the order parameter: the points within the soliton are mapped to the order parameter space $R=G / H$, while the points outside the soliton are mapped to the subspace $\tilde{R}=\tilde{G} / \tilde{H}$. The latter is restricted due to additional interaction which becomes important at large distances and which reduces the symmetry $G$ of the physical laws to its subgoup $\tilde{G}$. In the class of planar topological objects, domain walls are singular objects described by the group $\pi_{0}(R)$, while the smooth textures, such as planar solitons and Bloch walls, are described by the relative homotopy group $\pi_{1}(R, \tilde{R})$.

Analogues of such objects exist in topological matter. The role of singularities in the order parameter is played by the Green function's singularity in momentum space, such as nodes in the energy spectrum. For example, the analogue of a singular wall is an interface between the gapped bulk states, which contains gapless fermions, while the analog of a smooth wall is an interface, in which the Green's function has no singularity, i.e., within the nonsingular object the system remains fully gapped. The electronic structure of smooth textures is described by the relative homotopy groups $\pi_{n}(R, \tilde{R})$ which deal with two manifolds: the space $R$ of the Green's function within the texture and its subspace $\tilde{R}$ outside the texture. Outside the texture the space of the Green's function is restricted due to some symmetry in bulk, while within the texture this symmetry is violated or is spontaneously broken. Examples of the bulk symmetry are time reversal symmetry, spin rotation symmetry, crystal symmetry etc. Application 
of the RHG to classification of topological media has been also discussed in 12 .

In this paper we consider smooth interfaces which separate the bulk states of time reversal invariant insulators and fully gapped superfuid systems, their momentum-space topological invariants and the related quantum numbers. The relative homotopy group describing the fully gapped interfaces in the $d$-dimensional topological media is $\pi_{d+2}(R, \tilde{R})$.

\section{DOMAIN WALLS AND SOLITONS IN 3D TOPOLOGICAL MEDIA}

Let us consider examples of the singular and continuous interfaces in the vacuum described by Hamiltonian used in relativistic QFT:

$$
\hat{H}=\tau_{3} \boldsymbol{\sigma} \cdot \mathbf{p}+\tau_{1} m_{1}+\tau_{2} m_{2} .
$$

It represents a particle with a complex mass $m=$ $m_{1}+i m_{2}$. For real mass, $m_{2}=0$, the Hamiltonian obeys time reversal symmetry and anticommutes with the matrix $\tau_{2}$. The vacuum of this fermionic field is described by the 3D topological charge [13]

$N^{K}=\frac{1}{4 \pi^{2}} \operatorname{tr}\left[K \int_{\mathrm{BZ}} d^{3} k \mathcal{H}^{-1} \partial_{\left[k_{x}\right.} \mathcal{H} \mathcal{H}^{-1} \partial_{k_{y}} \mathcal{H} \mathcal{H}^{-1} \partial_{\left.k_{z}\right]} \mathcal{H}\right]$

Here the momentum space integral is over $\mathbb{R}_{3}$ in translational invariant systems, and over the Brillouin zone in crystals; $K$ is the matrix anticommuting with $\mathcal{H}$, which in a given case is $K=\tau_{2}$; we use antisymmetrization $f_{[i j \ldots]}=\frac{1}{n !} \sum_{S_{n}}(-1)^{P} f_{P i P j \ldots}$ over $n$ indices. The invariant (2) is valid also for time reversal invariant superfluids/superconductors, such as ${ }^{3} \mathrm{He}-\mathrm{B}$. For the Hamiltonian (11) with $m_{2}=0$ one has $N^{K}=m_{1} /\left|m_{1}\right|$. The interfaces - domain walls and solitons - are described by the coordinate dependent mass

$$
\hat{H}=\tau_{3} \boldsymbol{\sigma} \cdot \mathbf{p}+\tau_{1} m_{1}(z)+\tau_{2} m_{2}(z),
$$

where $z$ is the coordinate normal to the plane of the interface.

Singular walls: The singular interface is the domain wall separating the bulk states with different values of $N^{K}$, within which the symmetry $K$ is obeyed. For the Hamiltonian (1) this means that the mass remains real throughout the interface, $m_{2}(z) \equiv 0$, i.e., the time reversal symmetry is obeyed for the whole interface, while the mass $m_{1}$ crosses zero and changes sign within the interface, $m_{1}(-\infty)=-m_{1}(\infty)$. Since the bulk states have different topological charges, they cannot be connected adiabatically, and thus the domain wall necessarily contains gapless fermion zero modes. That is why such interface is considered as singular. It is the analog of the domain wall in ferromagnets in which magnetization crosses zero value. The singular interfaces and the gapless fermion modes inside them have been discussed for superfluid ${ }^{3} \mathrm{He}-\mathrm{B}$ in $[14,15$. On the topological and non-topological kinks and domain walls in Grand Unified Theories (GUT), see the book by Vachaspati [16].

Solitons and nonsingular walls: The nonsingular interface is obtained when the mass becomes complex within the interface, $m_{2}(z) \neq 0$. The time reversal symmetry is violated within the smooth interface and the spectrum becomes fully gapped everywhere. The interface where the phase of $m_{1}+i m_{2}$ changes by $\pi$ is the analog of the Bloch and Neel domain walls in ferromagnets, where the magnetization is nowhere zero and the orientation of the magnetization continuously changes across the wall. It is also the analogue of the topological soliton in ${ }^{3} \mathrm{He}-\mathrm{A}$ and ${ }^{3} \mathrm{He}-\mathrm{B}$. Contrary to the singular interfaces where the symmetry is restored in the core, in continuos interfaces the symmetry is smaller than that outside the interface. In relativistic theories, such interfaces have been considered by Wilczek [17] and also discussed in the book 16. Wilczek paid attention to the difference between the singular configuration which has gapless modes, and continuous configuration, which is fully gapped. For the gapless vacua in GUT this means that there are more massless particles outside such an interface than inside it 16 .

\section{5-FORM INVARIANT FOR SMOOTH INTERFACE IN 3D TOPOLOGICAL MEDIA}

In odd space dimension the continuous texture can be described by the topological invariant which characterizes the group $\pi_{d+2}(R, \tilde{R})$. For space dimension $d=3$ one has the group $\pi_{5}(R, \tilde{R})$ with the invariant

$$
\begin{aligned}
N_{5}=\frac{1}{4 \pi^{3} i} \operatorname{tr} \int_{\mathrm{BZ}} d^{3} k \int_{-\infty}^{\infty} d z \int_{-\infty}^{\infty} d \omega \\
\qquad \mathcal{G} \partial_{\left[k_{x}\right.} \mathcal{G}^{-1} \mathcal{G} \partial_{k_{y}} \mathcal{G}^{-1} \mathcal{G} \partial_{k_{z}} \mathcal{G}^{-1} \mathcal{G} \partial_{\omega} \mathcal{G}^{-1} \mathcal{G} \partial_{z]} \mathcal{G}^{-1}
\end{aligned}
$$

where $z$ is coordinate across the interface. This invariant in terms of the quasiclassical matrix Green's function $\mathcal{G}(\mathbf{k}, z, \omega)$ is applicable to different $d=3$ systems such as the 3D topological insulators and superfluid ${ }^{3} \mathrm{He}-\mathrm{B}$. The 5 -form integrals for topological media in terms of Green's functions have been discussed in Refs. [6, 18, 7]. The combined momentum space and real space topology has been applied for description of the singular topological defects and interfaces within the topological media and fermion zero modes in these ob- 
jects, see [19, 20, 14, 6, 21, 18, 22, and now we discuss this for continuous textures. For general continuous textures, $N_{5}$ may take any value, but it becomes integer or half-integer for appropriate boundary conditions when the bulk states at $z=\infty$ and $z=-\infty$ coincide, or are connected by symmetry.

As an example, consider Hamiltonian (3) with $m_{1}(z)=M_{1} \cos \phi(z)$ and $m_{2}(z)=M_{2} \sin \phi(z)$, where $\phi(z)$ changes from 0 to $\pi n$ across the interface. The gap is finite everywhere, and thus the integral (4) is welldefined and equals $N_{5}=n / 2 \operatorname{sign}\left(M_{1} M_{2}\right)$. For $n=2 k$, the states are the same on two sides of the soliton and $N_{5}$ is an integer. For $n=(2 k+1)$, the bulk states have opposite sign of mass $m_{1}$ and the invariant $N_{5}$ is a half integer. If time reversal symmetry is not obeyed in the bulk, the integral may take arbitrary values.

It is important that the invariant $N_{5}$ is determined both by the states of bulk topological matter outside the texture and by the internal structure of the texture. This invariant is expressed in terms of the Green's function, and thus is robust to perturbations such as interactions. In interacting systems the single-particle Hamiltonian, such as that which enters (2), is the secondary object. It is the effective Hamiltonian which belongs to the same topological class as the original interacting system, and thus can be adiabatically obtained from the interacting system. For example, one can consider the inverse Green's function at zero frequency as effective Hamiltonian, $\mathcal{H}_{\mathrm{eff}}(\mathbf{k})=\mathcal{G}^{-1}(\omega=0, \mathbf{k})$.

The invariant $N_{5}$ can be also applied to 3D topological insulators. Let us consider the model Hamiltonian for a TRI insulator (see e.g. [23]):

$$
\mathcal{H}=\gamma_{\mu} n_{\mu}(\mathbf{k}), \gamma_{0}=\tau_{1}, \gamma_{i}=\sigma_{i} \tau_{3}
$$

where $n_{\mu}$ is a 4 -vector and in a relativistic theory the matrices must be multiplied by $\tau_{1}$ to get the conventional $\gamma$-matrices. The particular 4 -vector discussed in [23] is:

$\mathcal{H}=-\lambda \tau_{3}\left(\sigma_{x} \sin k_{x}+\sigma_{y} \sin k_{y}+\sigma_{z} \sin k_{z}\right)+\tau_{1} m_{1}(\mathbf{k})$,

where $m_{1}(\mathbf{k})=M_{1}-t\left(\cos k_{x}+\cos k_{y}+\cos k_{z}\right)$. Inside the texture one has

$$
\begin{aligned}
& \mathcal{H}_{\text {texture }}=-\lambda \tau_{3}\left(\sigma_{x} \sin k_{x}+\sigma_{y} \sin k_{y}+\sigma_{z} \sin k_{z}\right) \\
& +\tau_{1} m_{1}(\mathbf{k}, z)+\tau_{2} m_{2}(\mathbf{k}, z) .
\end{aligned}
$$

One may choose, for example, the following texture: $m_{1}(\mathbf{k}, z)=M_{1} \cos \phi(z)-t\left(\cos k_{x}+\cos k_{y}+\cos k_{z}\right)$ and $m_{2}(\mathbf{k}, z)=M_{2} \sin \phi(z)$, with $\phi$ changing from 0 to $\pi n$ across the interface. For $2 t<\left|M_{1}\right|<3 t$ and large enough $\left|M_{2}\right|$, one obtains $N_{5}=n / 2 \operatorname{sign}\left(M_{1} M_{2}\right)$.

\section{5-FORM INVARIANT, $\theta$-TERM AND QHE}

For the 3D insulators the effects similar to those in axion QED take place. A $\theta$-term in the electromagnetic action has been proposed for the time reversal invariant (TRI) insulators, see, e.g., [24, 25, 23]:

$$
S=\frac{e^{2}}{32 \pi^{2}} \varepsilon^{\alpha \beta \mu \nu} \int d^{4} x \theta F_{\alpha \beta} F_{\mu \nu}=\frac{e^{2}}{4 \pi^{2}} \int d^{4} x \theta \mathbf{E} \cdot \mathbf{B} .
$$

In bulk insulators, $\theta$ is space-time constant, and this term does not make sense, since the action becomes a total derivative. Moreover, the $\theta$-term violates time reversal invariance and its application to TRI systems is tricky, though in a periodic space-time the situation is clearer [26]. However, all these problems vanish when we discuss the properties of a smooth texture within which the time reversal invariance is violated. While the parameter $\theta$ itself is ill-defined, the Hall conductivity in the plane of the interface is a well defined quantity, though formally according to (8) it can be related to the change of $\theta$ across the interface (see [27]):

$$
\frac{\sigma_{x y}}{\sigma_{\mathrm{H}}}=\frac{\theta(+\infty)-\theta(-\infty)}{2 \pi}, \sigma_{\mathrm{H}}=\frac{e^{2}}{h},
$$

Using the gradient expansion of the action, the Hall conductivity in the interface is expressed in terms of the invariant $N_{5}$ :

$$
\frac{\sigma_{x y}}{\sigma_{\mathrm{H}}}=N_{5} .
$$

Applying this to the Hamiltonian (3), where $\theta$ is related to the complex mass, $m_{2} / m_{1}=\tan \theta$ [17], for a texture where $m_{2}$ changes from $-m_{0}$ to $m_{0}$, one obtains

$$
\begin{aligned}
\frac{\sigma_{x y}}{\sigma_{\mathrm{H}}}= & \frac{1}{4 \pi^{3} i} \operatorname{tr} \int_{\mathrm{BZ}} d^{3} k \int_{-m_{0}}^{m_{0}} d m_{2} \int_{-\infty}^{\infty} d \omega \\
& \mathcal{G} \partial_{\left[k_{x}\right.} \mathcal{G}^{-1} \mathcal{G} \partial_{k_{y}} \mathcal{G}^{-1} \mathcal{G} \partial_{k_{z}} \mathcal{G}^{-1} \mathcal{G} \partial_{\omega} \mathcal{G}^{-1} \mathcal{G} \partial_{\left.m_{2}\right]} \mathcal{G}^{-1} .
\end{aligned}
$$

This transforms to the integer-valued $N_{5}$ in the limit of large $m_{0}$. The same is applied for the more general Hamiltonian $\mathcal{H}=\gamma_{\mu} n_{\mu}(\mathbf{k})+\gamma_{5} m_{2}, \gamma_{5}=\tau_{2}$.

According to (10), the Hall conductivity is determined both by the properties of bulk states outside the interface and by the internal structure of the interface. For the texture inside a TRI topological insulator, i.e., in the system which is TRI at $z= \pm \infty$, the Hall conductivity is quantized. Note that in this system the integral $N_{5}$ is a topological invariant, which belongs to the group $\mathbb{Z}$, i.e., it can take any integer or half-integer value, as distinct from the $\mathbb{Z}_{2}$ nature of the bulk insulator, where the parameter $\theta$ is ill defined. In other words, the group $\pi_{5}=\mathbb{Z}$ with its invariant $N_{5}$ describes the topology of the solitons within the topological insulator, rather than the insulators themselves. 


\section{1D SKYRMIONS AND THEIR TOPOLOGICAL AND QUANTUM NUMBERS}

The relative homotopy group $\pi_{3}(R, \tilde{R})$ with the 3 -form integral $N_{3}$ in terms of the Green's function $\mathcal{G}\left(\omega, k_{x}, x\right)$

$$
\begin{array}{r}
N_{3}=\frac{1}{4 \pi^{2}} \operatorname{tr} \int_{-\infty}^{\infty} d x \int_{-\infty}^{\infty} d \omega \int_{\mathrm{BZ}} d k_{x} \\
\mathcal{G} \partial_{\left[k_{x}\right.} \mathcal{G}^{-1} \mathcal{G} \partial_{\omega} \mathcal{G}^{-1} \mathcal{G} \partial_{x]} \mathcal{G}^{-1}
\end{array}
$$

describes 1D skyrmions in 1D gapped topological systems. These solitons have quantum numbers such as fermionic charge and quantized electric charge [28, 29, 30. In general these charges are expressed in terms of the topological charge $N_{3}$. Let us now consider the electric charge. Typically this charge is related to $1 \mathrm{D}$ $\theta$-term in the action,

$$
S=\frac{1}{2 \pi} \int d x d t \theta \varepsilon^{\alpha \beta} \partial_{\alpha} A_{\beta} .
$$

Again, this $\theta$-term does not make much sense for constant $\theta$, but it becomes meaningful for the inhomogeneous order parameter within the soliton where the time reversal symmetry is violated. Assuming $A_{0}$ is constant in space, one obtains the solitonic electric charge:

$$
\begin{gathered}
S=\frac{1}{2 \pi} \int d x d t \partial_{x} \theta A_{0}=\frac{1}{2 \pi} \int d t(\theta(+\infty)-\theta(-\infty)) A_{0}, \\
q=\frac{\theta(+\infty)-\theta(-\infty)}{2 \pi} .
\end{gathered}
$$

As distinct from the ill-defined $\theta$, the fermionic charge of the smooth structure is well defined. It is expressed in terms of the Green's function, and - using the gradient expansion - in terms of the quasiclassical Green's function $\mathcal{G}\left(\omega, k_{x}, x\right)$ :

$$
\begin{aligned}
q= & \frac{1}{4 \pi^{2}} \operatorname{tr} \int d x \int_{-\infty}^{\infty} d \omega \int_{\mathrm{BZ}} d k_{x} \\
& \mathcal{G} \partial_{\left[k_{x}\right.} \mathcal{G}^{-1} \mathcal{G} \partial_{\omega} \mathcal{G}^{-1} \mathcal{G} \partial_{x]} \mathcal{G}^{-1}
\end{aligned}
$$

which is the invariant $N_{3}$. Thus one obtains the general relation between the Green's function topological invariant $N_{3}$ characterizing the smooth structure and its fermionic charge $q$ :

$$
q=N_{3}
$$

Eq. (17) is analogous to equation (10) for quantization of Hall conductivity in the smooth interface within the 3D TRI topological insulators. It is valid for interacting systems as well.
Consider as an example the soliton in the following $1 \mathrm{D}$ system 24

$$
\hat{H}=\tau_{3} p_{x}+\tau_{1} m_{1}+\tau_{2} m_{2},
$$

which is time reversal invariant for $m_{2}=0$. For the soliton with $m_{1}(x)=M \cos \varphi(x), m_{2}(x)=M \sin \varphi(x)$, where $\varphi$ is going from 0 to $\pi n$, one obtains $N_{3}=n / 2$ and thus the charge of this soliton must be $q=n / 2$. The fermion number of the domain wall with $m_{2}=0$, $m_{1}(+\infty)=-m_{1}(-\infty)$ has been discussed by Jackiw and Rebby [28, who got the fermionic number $1 / 2$, i.e. $q= \pm 1 / 2$. This agrees with the topological charge $N_{3}= \pm 1 / 2$ of the soliton obtained by softening of the domain wall when the imaginary mass is added. Such softening does not change the boundary conditions at infinity and thus the fermionic charge may change only by integer number - the number of fermions.

\section{DISCUSSION}

We found a connection between topological invariants describing the smooth textures inside the topological media and their quantum numbers. We considered only one type of textures in $(1+1) \mathrm{D}$ and $(3+1) \mathrm{D}$ media. The other types of the continuous topological objects in topological matter are also possible. The relative homotopy group $\pi_{5}(R, \tilde{R})$ with the 5 -form integral $N_{5}$ in (12) in terms of the Green's function $\mathcal{G}\left(\omega, k_{x}, k_{y}, x, y\right)$

$$
\begin{aligned}
N_{5}=\frac{1}{4 \pi^{3} i} & \operatorname{tr} \int_{\mathrm{BZ}} d^{2} k \int d^{2} x \int d \omega \\
& \mathcal{G} \partial_{\left[k_{x}\right.} \mathcal{G}^{-1} \mathcal{G} \partial_{k_{y}} \mathcal{G}^{-1} \mathcal{G} \partial_{\omega} \mathcal{G}^{-1} \mathcal{G} \partial_{x} \mathcal{G}^{-1} \mathcal{G} \partial_{y]} \mathcal{G}^{-1},
\end{aligned}
$$

describes the 2D skyrmions in 2D gapped topological systems, such as ${ }^{3} \mathrm{He}-\mathrm{A}$ and planar phase. The quantum numbers of $2 \mathrm{D}$ skyrmions and the corresponding Chern-Simons terms in the action have been considered in [4, 5]. The relative homotopy group $\pi_{7}(R, \tilde{R})$ with the 7-form integral $N_{7}$ in terms of the Green's function $\mathcal{G}\left(\omega, k_{x}, k_{y}, k_{z}, x, y, z\right)$ describes the $3 \mathrm{D}$ skyrmions in the $3 \mathrm{D}$ gapped topological systems.

The mixed real-space and momentum-space topology can be applied for skyrmions and solitons in relativistic quantum field theories such as GUT, QCD, electroweak theory and theory of chiral and color quark matter. In particular, the $\theta$-term and axion electrodynamics 31 can be treated in the same manner as for ${ }^{3} \mathrm{He}-\mathrm{B}$ and TRI insulators, using integrals (44) and (2). The fermionic charges of skyrmions and other textures are related to the topological invariants expressed in terms of the fermionic propagator. 
This work is supported in part by the ERC (Grant No. 240362-Heattronics) and the Academy of Finland, Centers of excellence program 2006-2011.

\section{APPENDIX}

In this appendix, we show how Eq. (10) is obtained through the gradient expansion. By integrating out the fermions in the path integral, we obtain a current

$$
j^{\gamma}=\frac{\delta}{\delta A_{\gamma}} i \operatorname{Tr} \ln G=i e \operatorname{tr} \int \frac{d^{3} k d \omega}{(2 \pi)^{4}} G^{-1} \partial_{k_{\gamma}} G,
$$

which, at low energies, can be expanded in powers of gradients. Using Wigner transformed (or quasiclassical) Green functions $\mathcal{G}$ and the Moyal product rule, we obtain a gradient series for the Wigner transformed $G^{-1}$. From this series we will extract the part contributing to the current

$$
j^{\gamma}=\frac{e^{2}}{8 \pi^{2}} \varepsilon^{\alpha \beta \gamma \delta} F_{\alpha \beta} \partial_{\delta} \theta .
$$

This is second order in derivatives and we obtain

$$
\begin{aligned}
j^{\gamma}= & \frac{e^{2}}{2 i} F_{\alpha \beta} \operatorname{tr} \int \frac{d^{3} k d \omega}{(2 \pi)^{4}} \partial_{k_{\gamma}} \mathcal{G}\left\{\partial_{[\delta} \mathcal{G}^{-1} \partial_{\left.k_{\delta}\right]} \partial_{k_{\alpha}}\left(\mathcal{G} \partial_{k_{\beta}} \mathcal{G}^{-1}\right)\right. \\
& -\mathcal{G}^{-1} \partial_{k_{\alpha}} \mathcal{G} \partial_{k_{\beta}} \mathcal{G}^{-1} \partial_{[\delta} \mathcal{G} \partial_{\left.k_{\delta}\right]} \mathcal{G}^{-1} \\
& -\mathcal{G}^{-1} \partial_{[\delta} \mathcal{G} \partial_{\left.k_{\delta}\right]} \partial_{k_{\alpha}} \mathcal{G} \partial_{k_{\beta}} \mathcal{G}^{-1} \\
& -\partial_{k_{\beta}} \mathcal{G}^{-1} \partial_{k_{\alpha}}\left(\partial_{[\delta} \mathcal{G} \partial_{\left.k_{\delta}\right]} \mathcal{G}^{-1}\right) \\
& \left.-\frac{1}{2} \mathcal{G}^{-1} \partial_{k_{\beta}} \partial_{[\delta} \mathcal{G} \partial_{\left.k_{\delta}\right]} \partial_{k_{\alpha}} \mathcal{G}^{-1}\right\},
\end{aligned}
$$

where $\mathcal{G}$ is evaluated in zero external field. For a linear Hamiltonian we have $\partial_{k_{\alpha}} \partial_{k_{\beta}} \mathcal{G}^{-1}=0$ and finally obtain the action

$$
\begin{aligned}
S= & \frac{e^{2}}{16 \pi^{2}} \int d^{3} x d t \varepsilon^{\alpha \beta \gamma \delta} F_{\alpha \beta} A_{\gamma} \partial_{\delta} \theta \\
\partial_{\delta} \theta= & \frac{1}{2 \pi^{2} i} \operatorname{tr} \int_{\mathrm{BZ}} d^{3} k \int_{-\infty}^{\infty} d \omega \\
& \mathcal{G} \partial_{[\delta} \mathcal{G}^{-1} \mathcal{G} \partial_{k_{\lambda}} \mathcal{G}^{-1} \mathcal{G} \partial_{k_{\mu}} \mathcal{G}^{-1} \mathcal{G} \partial_{k_{\nu}} \mathcal{G}^{-1} \mathcal{G} \partial_{\left.k_{\rho}\right]} \mathcal{G}^{-1},
\end{aligned}
$$

which leads to Eq. (10).

In exactly the same fashion, we obtain Eq. (17). In this case the current of Eq. (20) is obtained from the first order gradient expansion and reads

$$
j^{\gamma}=\frac{e}{4 \pi^{2}} \operatorname{tr} \int_{-\infty}^{\infty} d \omega \int_{\mathrm{BZ}} d k_{x} \mathcal{G}^{-1}\left(\partial_{[\alpha} \mathcal{G} \partial_{k_{\alpha]}} \mathcal{G}^{-1}\right) \partial_{k_{\gamma}} \mathcal{G}
$$

1. K. Ishikawa and T. Matsuyama, Magnetic field induced multi component QED in three-dimensions and quantum Hall effect, Z. Phys. C 33, 41-45 (1986).
2. K. Ishikawa and T. Matsuyama, A microscopic theory of the quantum Hall effect, Nucl. Phys. B 280, 523-548 (1987).

3. T. Matsuyama, Quantization of conductivity induced by topological structure of energy-momentum space in generalized $\mathrm{QED}_{3}$, Progr. Theor. Phys. 77, 711-730 (1987).

4. G.E. Volovik and V.M. Yakovenko, Fractional charge, spin and statistics of solitons in superfluid ${ }^{3} \mathrm{He}$ film, J. Phys.: Cond. Matter 1, 5263-5274 (1989).

5. V.M. Yakovenko, Spin, statistics and charge of solitons in (2+1)-dimensional theories, Fizika (Zagreb) 21, suppl. 3, 231 (1989); cond-mat/9703195

6. G.E. Volovik, The Universe in a Helium Droplet, Clarendon Press, Oxford (2003).

7. Zhong Wang, Xiao-Liang Qi and Shou-Cheng Zhang, Topological order parameters for interacting topological insulators, Phys. Rev. Lett. 105, 256803 (2010).

8. M. Golterman, K. Jansen and D. Kaplan, Chern-Simons currents and chiral fermions on the lattice, Phys. Lett. B 301, 219-223 (1993); arXiv:hep-lat/9209003.

9. D.L. Bergman, Axion response in gapless systems, arXiv:1101.4233.

10. Hong Yao and Dung-Hai Lee, Topological insulators and topological non-linear $\sigma$-models, arXiv:1003.2230

11. V.P. Mineev and G.E. Volovik, Planar and linear solitons in superfluid ${ }^{3} \mathrm{He}$, Phys. Rev. B 18, 3197-3203 (1978).

12. A.M. Turner, Yi Zhang, R.S.K. Mong and A. Vishwanath, Quantized response and topology of insulators with inversion symmetry, arXiv:1010.4335.

13. G.E. Volovik, Topological invariants for Standard Model: from semi-metal to topological insulator, JETP Lett. 91, 55-61 (2010); arXiv:0912.0502.

14. M.M. Salomaa and G.E. Volovik, Cosmiclike domain walls in superfluid ${ }^{3} \mathrm{He}-\mathrm{B}$ : Instantons and diabolical points in (k,r) space, Phys. Rev. B 37, 9298-9311 (1988).

15. G.E. Volovik, Topological invariant for superfluid ${ }^{3} \mathrm{He}-\mathrm{B}$ and quantum phase transitions, JETP Lett. 90, 587-591 (2009).

16. T. Vachaspati, Kinks and Domain Walls: An Introduction to Classical and Quantum Solitons, Cambridge University Press (2006).

17. F. Wilczek, Two applications of axion electrodynamics, Phys. Rev. Lett. 58, 1799-1802 (1987).

18. M.A. Silaev and G.E. Volovik, Topological superfluid ${ }^{3} \mathrm{He}-\mathrm{B}$ : fermion zero modes on interfaces and in the vortex core, J. Low Temp. Phys, 161, 460-473 (2010), arXiv:1005.4672.

19. G.E. Volovik and V.P. Mineev, Current in superfluid Fermi liquids and the vortex core structure, JETP 56, 579-586 (1982).

20. P.G. Grinevich and G.E. Volovik, Topology of gap nodes in superfluid ${ }^{3} \mathrm{He}: \pi_{4}$ homotopy group for ${ }^{3} \mathrm{He}-\mathrm{B}$ disclination, J. Low Temp. Phys. 72, 371-380 (1988). 
21. J.C.Y. Teo and C.L. Kane, Topological defects and gapless modes in insulators and superconductors, Phys. Rev. B 82, 115120 (2010).

22. V. Gurarie, Single particle Green's functions and interacting topological insulators, arXiv:1011.2273.

23. G. Rosenberg and M. Franz, Witten effect in a crystalline topological insulator, arXiv:1001.3179.

24. Xiao-Liang Qi, Taylor L. Hughes, and Shou-Cheng Zhang, Topological field theory of time-reversal invariant insulators, Phys. Rev. B 78, 195424 (2008).

25. A.M. Essin, J.E. Moore and D. Vanderbilt, Magnetoelectric polarizability and axion electrodynamics in crystalline insulators, Phys. Rev. Lett. 102, 146805 (2009).

26. Kuang-Ting Chen and Patrick A. Lee, Topological Insulator and the $\theta$-vacuum in a system without boundaries, arXiv:1012.2084

27. M.Z. Hasan and J.E. Moore, Three-dimensional topological insulators, arXiv:1011.5462.

28. R. Jackiw and C. Rebbi, Solitons with fermion number $\frac{1}{2}$, Phys. Rev. D 13, 3398-3409 (1976).

29. W.P. Su, J.R. Schrieffer and A. Heeger, Solitons in polyacetylene, Phys. Rev. Lett. 42, 1698 (1979).

30. J. Goldstone and F. Wilczek, Fractional quantum numbers on solitons, Phys. Rev. Lett. 47, 986-989 (1981).

31. R.D. Peccei and H.R. Quinn, $C P$ conservation in the presence of pseudoparticles, Phys. Rev. Lett. 35, 1440 1442 (1977). 